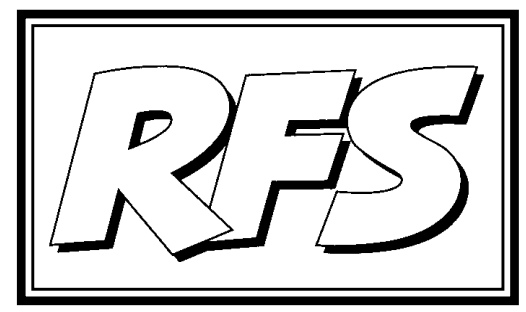

Revista de Fomento Social, 60 (2005), 463-490

\title{
La reforma de la administración andaluza y los desafíos de la segunda modernización
}

\section{Antonio J. PORRAS NADALES ${ }^{1}$}

(Palabras clave: Gobernanza, Comunidad Autónoma, Andalucía, Segunda MODERNIZACIÓN, ADMINISTRACIÓN PÚBLICA.

Key Words: Governance, Autonomous Administration, Andalusia, SeCond

Modernisation, Public Administration)

1 Catedrático de Derecho Constitucional. Universidad Pablo de Olavide (Sevilla). Con el apoyo del Grupo de Investigación sobre "Gestión Pública y Políticas Públicas", SEJ 373, del Plan Andaluz de Investigación. 


\section{La gestión pública ante un contexto histórico transformado}

Al cabo de más de dos décadas de proceso autonómico, los problemas relativos a la gestión pública en España comienzan a percibirse desde coordenadas bastante diferentes a las que presidieron sus etapas originales a comienzos de los ochenta. Frente a la impronta fuertemente "politizada" que caracterizó a la etapa fundacional, con una preocupación dominante orientada hacia la inmediata puesta en marcha de los servicios y competencias transferidas, la situación a comienzos del sigloXXI parece que debe abordarse desde unos parámetros sustancialmente transformados. Una transformación que afecta no solo al plano doctrinal (donde, en general, puede detectarse un desarrollo espectacular de nuevos paradigmas científicos, sobre todo a partir de la noción de "Nueva Gestión Pública") ${ }^{2}$, sino igualmente al contexto institucional, que ha estado sometido a una intensa evolución dentro de la cual las propias Comunidades Autónomas han desarrollado numerosos procesos de innovación y aprendizaje.

La primera de esas transformaciones podría situarse en torno a la creciente centralidad que ha adquirido la noción de gobernanza (del inglés "governance", aceptada ya como traducción castellana a partir de la versión del Libro Blanco de la Unión Europea de 2001), que responde a una larga inercia de preocupación por la eficacia de la acción de gobierno, implicando una superación del anterior y tradicional paradigma de la "gobernabilidad", que se reducía en última instancia a la mera estabilidad gubernamental. La noción de governance supone adicionalmente un conjunto de exigencias que se orientan hacia una proyección más eficaz de la acción pública, implicando tanto el desarrollo de políticas públicas desde entornos participados con presencia de redes sociales, como la aplicación de nuevas formas de gestión que rompen con los tradicionales paradigmas de la rutina y la verticalidad, propias del viejo modelo burocrático maxweberiano. Adicionalmente, las exigencias de la gobernanza parecen implicar también una cierta capacidad para progresar dentro de un emergente sistema de red, en base a claves de

2 M. Barzelay (1992), Breaking through Bureaucracy, Univ. California Press (trad. cast. FCE); (2001), The New Public Management. Improving Research and Policy Dialogue, Univ. California Press; G. MaJone (1989), Evidence, Argument and Persuasion in the Policy Process, Yale UP (trad. cast. FCE); R. HEIFETz (1997), Liderazgo sin respuestas fáciles, Barcelona, Paidos; D. OSBORNE y P. PLASTRIK (1998), La reducción de la burocracia, cinco estrategias para reinventar el gobierno, Barcelona, Paidos; M. H. Moore (1998), Gestión estratégica y creación de valor en el sector público, Barcelona, Paidos. 
cooperación y coordinación, que tienen particularmente en Europa su mejor campo de experimentación y desarrollo ${ }^{3}$.

En segundo lugar, esta innovación, que ahora procede fundamentalmente del contexto europeo, coincide con la propia secuencia evolutiva por la que discurre el proceso autonómico español: un proceso de dimensión histórica y de intenso impacto transformador donde parece emerger la evidencia de enfrentar una "nueva etapa", una vez superadas la fase constitutiva o maximalista inicial y también la segunda gran etapa que abarca el largo y conflictivo periodo transferencial o de "lucha por las competencias". Es cierto que la lógica conflictual, propia de este segundo periodo, ha generado una consistente inercia que condiciona la propia percepción general del proceso autonómico, que suele ser entendido como un interminable fenómeno de tensión anti-centro: los "flecos" pendientes que a veces ha dejado el proceso de transferencia de competencias, así como el propio retraso con que el Tribunal Constitucional viene resolviendo los conflictos de competencias, contribuyen a acentuar esta percepción inercial de tipo conflictivo, ocultando así la emergencia de una nueva etapa, donde el conflicto por las competencias parece comenzar a asumir un valor secundario frente a un nuevo desafío no menos decisivo: el de avanzar hacia un ejercicio eficiente de las mismas a través del desarrollo de políticas públicas eficaces ${ }^{4}$.

Pese a esta dinámica inercial, el cambio en el ciclo histórico se viene percibiendo de una forma generalizada traduciéndose en un proceso de reflexión orientado finalmente hacia la reforma de los Estatutos de Autonomía. Aunque la preocupación general por el nuevo proceso de reformas estatutarias parece inclinarse más bien hacia una reformulación general de las reglas de juego, sin embargo, desde nuestra perspectiva consideramos que tal evolución hacia una nueva etapa de desarrollo autonómico marcaría alternativamente una original novedad estratégica consistente en el paso de la fase de apogeo de la "política" a la creciente centralidad de las "políticas"; es decir, a un contexto donde el auténtico desafío deberá consistir en la capacidad para introducir nuevos enfoques y pautas de gestión pública en las respectivas esferas competenciales y organizativas, orientadas hacia

3 R. MAyntz (1999), "La teoria della governance. Sfide e prospettiva”, Rivista Italiana di Scienza Politica, $\mathrm{n}^{\circ}$ 1. Libro Blanco sobre la gobernanza de la UE, 2001, www.europa.eu.int.

4 A. J. Porras Nadales (2003), "Del proceso autonómico hacia un sistema de red. (Regiones y Comunidades Autónomas en los albores del siglo XXI)", Revista de Fomento Social, n 58 , pp. 35-59. 
la generación de respuestas eficaces por parte de la esfera pública ante el nuevo tipo de demandas que se suscitan desde la esfera social. Probablemente un tipo de innovación que estaría más próxima a las preocupaciones auténticas de los ciudadanos.

Esta línea de desarrollo no afecta tan sólo a la esfera de la propia organización pública, sino que tiene un impacto adicional sobre la opinión pública, reflejando una tendencia de evolución sociocultural donde los propios sujetos y colectivos afectados por las políticas públicas comienzan a asumir un papel más activo: las nuevas exigencias de calidad, participación, evaluación y control, pasan así a configurarse como un bloque de valores culturales que se traducen en un papel más activo y al mismo tiempo más crítico por parte de los ciudadanos, entendidos como usuarios de servicios públicos. Todo ello vendrá a incidir inexorablemente sobre las exigencias de autodesarrollo y aprendizaje de las propias organizaciones públicas.

Ahora bien, esta percepción del proceso de cambio histórico, que se proyectaría en una dimensión progresivamente globalizada, debe enfrentarse a los obstáculos que presentan las tendencias alternativas que impulsan hacia el anquilosamiento y el conservadurismo de unos modelos autonómicos de organización pública que, diseñados a imagen y semejanza del anterior estado centralista, han servido para reproducir y acentuar las más rancias tradiciones de la cultura burocrática preexistente ${ }^{5}$. En particular si tenemos en cuenta que los criterios de selección y encuadramiento del personal público han adolecido de fuertes dosis de politización y clientelismo, generando así una difusa cultura de resistencia al cambio, así como una proyección opaca y escasamente transparente de los mecanismos que conforman la "carrera" del personal público.

Por supuesto, este fenómeno de anquilosamiento histórico no es ajeno a la propia influencia del estado central, que no ha sido capaz hasta ahora de impulsar las necesarias sinergias de cambio: el fracaso de los proyectos de reforma de la administración que se pusieron en marcha a finales de los ochenta bajo el ministerio Almunia, ha dejado sin cubrir toda una gran tarea pendiente para el conjunto del sistema público del estado; y la posterior oleada de iniciativas "modernizadoras" tanto en la esfera central como en la

5 C. Ramió y M. SALVADOR (2002), "La configuración de las administraciones de las comunidades autónomas: entre la inercia y la innovación institucional" en J. Subirats y R. Gallego (ed.), Veinte años de autonomías en España. Leyes, políticas públicas, instituciones y opinión pública, Madrid, CIS, pp. 99-133. 
autonómica, ha venido operando predominantemente en circuitos periféricos o cosméticos del sistema público, sin alterar apenas el modelo nuclear y originario de administración. Incluso la puesta en marcha de algunas iniciativas sustanciales como las agencias reguladoras independientes, especialmente en el sector energético o financiero, no se ha traducido en resultados claramente positivos en términos de consolidación institucional ${ }^{6}$.

\section{Las premisas de una política pública de gestión pública}

Parece, en definitiva, que las claves evolutivas por las que ha discurrido el proceso autonómico español no permiten detectar con claridad la existencia de esa necesaria "percepción problemática" de la administración y del sector público, entendida como el núcleo argumentativo del que debería surgir un programa de reformas susceptible de traducirse en nuevos modelos organizativos capaces de generar una mayor eficiencia en la acción pública autonómica. Por el contrario, puede considerarse hasta ahora como dominante el paradigma tradicional, desde el cual se supone que toda acción de programación política se instrumentaliza mecánicamente y se lleva a cabo de forma más o menos automática a través de los mecanismos legales y del funcionamiento rutinario de la administración burocrática. Las deficiencias en la implementación, la falta de diseño organizativo, el retraso en los programas, el fracaso en el logro de objetivos, se siguen considerando desde la resignada óptica de lo inevitable: los medios o instrumentos de acción serían como un elemento dado, sometido a la inexorable lógica que impone la realidad existente, y en ningún caso un factor disponible, cuya modificación resulta cada día más urgente para avanzar en los desafíos históricos de la gobernanza.

Frente a esta óptica de resignación, cabe enfrentar en primer lugar la necesaria toma de conciencia de estar ante un auténtico "problema" de dimensión social, que afecta al modo como se definen e implementan los valores propios de una sociedad democrática a través de la acción de las esferas públicas: las claves argumentativas desde las cuales se evidencia, según la doctrina, la necesidad de poner en marcha una política pública orientada a transformar la propia gestión pública, se sitúan precisamente en torno a esta idea originaria consistente en la percepción como "problema"

6 K. Echavarría (2000), “La gestión pública a través de agencias: experiencias europeas”, en F. Longo y M. Zafra (coord.), Pensar lo público, Granada, Cemci. 
de esta deficiencia instrumental, de la que dependen las claves del éxito de toda acción pública. En un diseño esquemático, tal argumentación partiría de la interconexión entre un determinado diagnóstico de la realidad (que debe implicar necesariamente una cierta dimensión crítica de la misma, en la medida en que trata de detectar y definir un "problema"), y un tipo de respuesta al mismo que se traduciría en unas concretas orientaciones finalistas o programación de objetivos; las cuales, para ser llevados a cabo, requieren a su vez de ciertos instrumentos de acción. En rigor, toda formulación de una política pública debe responder coherentemente a esta secuencia argumentativa simplificada, que afectará finalmente a los propios instrumentos de la acción pública: diagnóstico, fines y medios. En la medida pues en que exista una efectiva percepción colectiva de este problema, surgiría la necesaria puesta en marcha de una política pública de gestión pública.

Debemos entender que tal percepción problemática tiene probablemente su principal campo estratégico de experimentación en la arena regional o autonómica, que es donde se han venido concentrando las principales líneas de evolución histórica del estado social, conforme a la conocida pauta de regionalización del mismo, evidenciada particularmente en la arena europea $^{7}$. Será por lo tanto en la esfera regional europea donde los elementos de argumentación y evidencia que deberían concretarse en una política pública de gestión pública, adquirirán una mayor centralidad estratégica: lo que implica la necesidad de hacer frente a las reiteradas sinergias que contribuyen al mantenimiento del conservadurismo y de los modelos tradicionales de organización y funcionamiento de la administración autonómica.

Para el caso de Andalucía podemos pues tratar de situar tales sinergias de carácter conservador alrededor de estos tres elementos argumentativos centrales: el diagnóstico, los objetivos y el soporte instrumental.

A) El diagnóstico. La visión de los diagnósticos sobre la realidad andaluza parece desde hace tiempo inmersa en una clara visión ultraoptimista de la realidad, que se suele proyectar en una clave de tipo temporal o cronológico a través del postulado de que "todo tiempo pasado fue peor". En una perspectiva histórica confluyen aquí dos elementos, por una parte la larga memoria de la posguerra desde la cual el acceso a la autonomía se entendía a partir de mediados de los años setenta no sólo como un componente esencial del retorno a la democracia sino como

F. SCHARPF (1996), “Unione europea e welfare state nazionali”, Rivista Italiana di Scienza Politica, $\mathrm{n}^{\mathrm{o}} 1$. 
un instrumento político de superación del subdesarrollo económico y el atraso histórico ${ }^{8}$; por otra, el impacto negativo del largo flujo de emigración desde los años sesenta, entendido como una pérdida inexorable de tejido social sustantivo, que contribuyó a acentuar la sensación de victimalidad espacial y la conciencia de subdesarrollo que impulsó la fuerte demanda autonómica ${ }^{9}$. Proyectado hacia las coordenadas del presente, tal diagnóstico encuentra su expresión en la llamada "paradoja de la satisfacción" ${ }^{10}$, reflejo de una visión optimista de la realidad que, sin embargo, se proyecta en un sentido dicotómico en torno a la curva de edad (es decir, es una visión característica, sobre todo, de las personas de mayor edad). En última instancia, los diagnósticos sobre la realidad andaluza se diseñan pues desde la ausencia de cualquier perspectiva crítica de la realidad, neutralizando así cualquier potencial problemático del que pueda deducirse la necesidad de hacer frente a reformas o innovaciones sustantivas en la realidad existente.

B) Los objetivos. En relación con la formulación de horizontes finalistas, en Andalucía predominaría hasta ahora la concepción tradicional, vinculada al apogeo del estado de partidos, según la cual se entiende que los objetivos de la acción pública se determinan exclusivamente en la arena política, o más bien "político-electoral". Por lo tanto, no serían una esfera disponible para el gestor público, ni siquiera en su condición de directivo; ni en principio tendrán tampoco una clara conexión con ningún tipo de conocimiento técnico-científico. Por lo tanto, su sistema de evaluación reposa en todo caso en la decisión ciudadana a través de las urnas, y no en una rigurosa evaluación de resultados. Tan sólo en un periodo relativamente reciente, en torno a la experiencia del "Foro Andalucía en el Nuevo Siglo", aparece por primera vez un cierto protagonismo del conocimiento experto, aunque dentro de una programación muy abierta y relativamente dispersa.

8 A. J. Porras Nadales (2002), "El marco político de la transición en Andalucía”, Congreso sobre la transición en Andalucía, Universidad de Cádiz, (en prensa).

9 Ibid. Cfr. también J. A. LacomBa (1999), "Historia e identidad: de la historia en Andalucía a la historia de Andalucía”, en J. Hurtado, E. Fernández de Paz, Cultura Andaluza, Ayuntamiento y Universidad de Sevilla. J. CAzorla (1965), Factores de la estructura socioeconómica de Andalucía Oriental, Granada, Caja de Ahorros de Granada.

10 M. Pérez Yruela (2002), "Para una nueva teoría de Andalucía: cambio y modernización en la sociedad andaluza", en E. Moyano Estrada y M. Pérez Yruela (eds.) (2000), La Sociedad Andaluza, Córdoba, IESA. 
C) Finalmente, un mismo grado de relativa indisponibilidad planea sobre la esfera instrumental, donde predomina el apriorismo de que los medios o elementos instrumentales de la acción reposan sobre la esfera estable y predefinida del sistema legal-burocrático, que constituye en rigor un sistema preestablecido y por lo tanto no disponible por el propio gestor público. Siguiendo en general la filosofía propia del periodo de apogeo del estado social, se entiende como máximo que, en todo caso, la instrumentación o implementación de las políticas públicas dependerá de la cantidad de recursos financieros públicos que se asignen a las mismas, lo que estará condicionado pues, en última instancia, por claves esencialmente políticas ${ }^{11}$.

Se nos dibujaría así un panorama conservador, carente de perfiles críticos en su diagnóstico o visión de la realidad, y caracterizado por una situación de indisponibilidad en lo que respecta a fines y medios por parte de la esfera de la propia gestión pública. De este modo, las premisas argumentativas desde donde teóricamente podría emerger la "necesidad" de una política pública de gestión pública, parecen bastante diluidas.

En este contexto, la figura del gestor público (entendida como impulsor o protagonista del nuevo enfoque de la acción pública) aparecería finalmente estrangulada o aprisionada entre dos grandes circuitos que, aparentemente, suministrarían soportes de racionalidad suficientes para la acción (aunque de una forma completamente ajena al propio circuito de la gestión pública): por una parte el imperio de la política, por otra el imperio del derecho. (A) Desde el circuito de la política, las claves finalistas que condicionan la acción pública vendrían determinadas por el eje programático-electoral validado por las urnas: y en consecuencia, serán en último término los políticos, la clase gobernante, quienes se encarguen de dirigir la acción, definiendo los objetivos y nombrando para ello a los cargos directivos en base a criterios de pura confianza política. (B) Por su parte, desde el circuito del derecho, el paradigma del imperio de la ley (promulgada en el marco de las orientaciones finalistas previstas tanto en la Constitución como en el Estatuto) reiteraría el arcaico diseño de una función "ejecutiva" entendida como mera

11 En un sentido alternativo al que constituye uno de los desafíos fundamentales de la nueva gestión pública, el que formulara paradigmáticamente AL Gore (1993), en el sentido de conseguir una administración que funcione mejor y que cueste menos, cfr. su From Red Tape to Results: Creating a Government that Works Better ad Costs Less, Washington, Nueva York, Times Books. 
labor mecánica, asignada a una burocracia subordinada a la ley y al derecho y controlada por los jueces; en consecuencia, la esfera del gobierno y la administración carecería en rigor de autonomía estratégica suficiente para enfrentar reformas en profundidad de los modelos existentes o para poner en marcha nuevos modelos de acción pública.

En este contexto, ninguna de las nuevas categorías o valores que, teóricamente, cabe esperar de una moderna gestión pública, como la capacidad organizativa, la habilidad negociadora, la capacidad gestora, el liderazgo público, o el propio conocimiento especializado, tendrían un cauce adecuado para desarrollarse, predominando alternativamente los valores de la subordinación y la lealtad al poder político, o la mera rutina burocrática adecuada a pautas legales.

\section{El desarrollo de la nueva gestión pública en las democracias contem- poráneas}

Semejante percepción de la realidad provoca un inevitable alejamiento de algunos de los circuitos de innovación por los que discurre al nivel comparado el intervencionismo público desde hace ya algunas décadas. Recordemos que desde la perspectiva de la evolución del estado social, la preocupación fundamental se centra en la cuestión de la eficacia de los instrumentos que canalizan la acción pública: una preocupación frecuentemente desbordada ante el apogeo del mercado y las amenazas de privatización y desregulación del propio sector público. Tal exigencia de eficacia impacta sobre los tradicionales modelos de organización propios del Estado de Derecho, que nacieron del contexto histórico liberal (abstencionista), y que se han venido readaptando -en un ambiente de dificultades, bloqueos y anquilosamientos difusos- a las nuevas exigencias que, desde mediados del siglo XX, impone el modelo constitucionalizado del estado social.

Frente a la tradicional percepción estática del Estado de Derecho, la propia naturaleza del intervencionismo público hace del estado social un modelo en constante proceso de evolución y cambio: pero las pautas de este proceso adaptativo seguido por el estado social han debido enfrentarse en España al lógico retraso histórico que impuso la dictadura franquista. De tal modo que algunas de las iniciativas de innovación y reforma de los aparatos públicos que se han venido poniendo en marcha en las democracias occidentales durante las últimas décadas, siguen adoleciendo de un considerable déficit de percepción, cuando no de un claro desconocimiento en nuestra tierra. 
En todo caso habría que recordar que la evolución del estado social no se limita exclusivamente a la esfera instrumental o prestadora, sino que afecta al conjunto del sistema político: en su percepción más nuclear, el diagnóstico se situaría en torno a la denominada "crisis de reflexión" del centro del sistema, entendida desde Luhmann ${ }^{12}$ como una incapacidad del circuito político central (el eje mayoría política-gobierno) para responder de forma racional y unificada al conjunto creciente, complejo y contradictorio de demandas del sistema. Esto implicaría dos consecuencias. Primera, que las esferas centrales gubernamentales estarían dejando de ser en rigor instrumentos "planificadores" (como en alguna ocasión se llegó a soñar durante la década de los sesenta), con capacidad para unificar racionalmente el conjunto de la acción pública desde una perspectiva instrumental o mecanicista ${ }^{13}$, para pasar a convertirse en meros circuitos "orientadores" de la acción. Un tipo de cambio que se percibe con claridad en el modo como se diseñan en la práctica los programas de gobierno en los procedimientos de investidura, entendidos como meras formulaciones de líneas generales de acción. Y segunda consecuencia, que lógicamente el grueso de la acción y la responsabilidad pública se desplazaría ahora hacia las esferas periféricas del sistema: lo que nos conecta de forma inmediata con la expansión del regionalismo y la recomposición en clave regional europea del propio estado social ${ }^{14}$. Ahora bien, este desplazamiento de la responsabilidad intervencionista sobre la periferia del sistema no afecta solamente al circuito territorial, sino igualmente a la periferia "organizativa", es decir, al sector público en su conjunto, implicando una mayor responsabilidad en el desarrollo de la acción intervencionista, ante un contexto donde los mandatos procedentes de la esfera política ya no están suficientemente explicitados (puesto que, en rigor, se limitarían a simples orientaciones más o menos difusas, cuando no a puros posicionamientos cosméticos).

Por lo que respecta al proceso general de introducción de las nuevas pautas de gestión pública al nivel comparado, se ha destacado en primer lugar el

12 N. Luhmann (1982), Teoría Política en el Estado del Bienestar, Madrid, Alianza.

13 Para una crítica de las concepciones instrumentalistas del estado, cfr. C. Offe (1988), Partidos políticos y nuevos movimientos sociales, Madrid, Sistema.

14 F. ScharpF, "Unione europea e welfare state nazionali”, Rivista Italiana di Scienza Politica, cit. También más en general, M. A. APARICIO (coord.) (2001), Derechos Constitucionales y Formas Políticas. Actas del Congreso sobre derechos constitucionales y Estado autonómico, Barcelona, Cedecs. 
papel de avanzadilla que asumieron desde mediados de los ochenta ciertos países de tradición anglosajona como Nueva Zelanda, Australia o Inglaterra ${ }^{15}$ cuyos sistemas legales y modelos culturales dominantes se caracterizan en general por una mayor ductilidad y mejor adaptabilidad al cambio histórico. Una segunda oleada posterior de reformas afectaría desigualmente a otras zonas como los países nórdicos, Alemania, o bien a otras áreas geopolíticas como Brasil o México. En cambio, algunos de los países vinculados o bien a la tradición católica o bien a la "cultura" administrativista europea continental, como Italia o Francia, presentarían procesos más tardíos o limitados en el desarrollo de políticas de gestión pública.

Los análisis comparativos han ido señalando paralelamente todo un conjunto de factores que de forma variable explican el mayor o menor grado de éxito de estos programas: desde la influencia de las distintas formas de estado o de gobierno, la presencia de modelos de ejecutivos de tipo mayoritarios o de tipo consensual, el tipo de relaciones que se producen entre los ministros y los altos burócratas (o, más en general, entre la clase política y la clase burocrática), el tipo de cultura administrativa dominante en cada caso, o incluso la existencia de canales de asesoramiento de políticas públicas.

Hasta ahora un balance realista del caso español nos confirmaría su inevitable aproximación a los países de nuestra área, es decir, a los casos de Francia o Italia, donde el impacto de las transformaciones del sector público parece ser todavía bastante limitado. Tras el fracaso de los proyectos puestos en marcha por el ministro Almunia a finales de los ochenta (a consecuencia de la emergencia de los primeros grandes casos de corrupción política), las líneas de avance en este terreno se han situado más bien en la arena de la "modernización" administrativa y no tanto en la introducción de grandes programas de reforma: lo que significa la incorporación difusa de las nuevas tecnologías, o de programas de apertura general de la administración al ciudadano (puntos de información, ventanillas únicas, etc.). Con la dificultad añadida que supone el desarrollo del estado autonómico al implicar una pluralidad de posibilidades de innovación administrativa y una gran diversidad de experiencias prácticas.

La experiencia comparada nos demuestra pues que los impulsos de reforma general de la administración y de introducción de una política de gestión

15 Cfr. C. Pollit y G. Bouckaert (2000), Public Management Reform: a Comparative Analysis, Oxford. Oxford University Press. 
pública exigen toda una amplia y compleja movilización de recursos políticos y estratégicos (además de financieros en su caso), ante la cual las burocracias y en general las organizaciones públicas siempre tienen la opción alternativa de dejarse caer indolentemente del lado perverso de la no-acción, limitándose al simple mantenimiento de las rutinas y al deficiente cumplimiento de sus responsabilidades y programas, bajo el resignado designio de lo inexorable. Del mismo modo que la esfera política central puede también repetir hasta la saciedad su estrategia de interferencia sobre la acción pública, reiterando así el conocido diagnóstico de la "crisis de reflexión", y operando en consecuencia en una lógica "repolitizadora" del sistema, o de "colonización" de la política sobre la burocracia y sobre el propio tejido social organizado.

Pero frente a estas derivaciones perversas, cabrá plantear alternativamente el auténtico problema central, consistente en cómo asegurar un funcionamiento más eficaz de las organizaciones públicas en el contexto evolucionado del estado social intervencionista. Es aquí donde adquiere su verdadera significación la aparición y desarrollo de todo un discurso científico sobre la "nueva gestión pública", entendida como una forma dinámica y congruente de responder a esta compleja serie de exigencias.

La puesta en práctica de este proceso global, considerado como el modo de acción propio del estado social avanzado, o estado postsocial ${ }^{16}$, significará en consecuencia una nueva forma de entender el papel instrumental de la administración claramente superadora del modelo rutinario y estable del tradicional paradigma maxweberiano y sus implicaciones de tipo legal-racional $^{17}$. En este caso, la noción de implementación y la generalización del enfoque del policy analysis vendrán a sustituir a la vieja visión de la administración concebida como una instancia limitada a la mera "ejecución" de los mandatos legales, implicando pues una necesaria autonomía creativa tanto en términos de organización como de acción. Igualmente la autonomía estratégica de que deberá gozar el gestor, en su condición de directivo público, permite entender que la gestión pública deberá desempeñar ahora una función a la que cabe atribuir en cierto sentido un carácter "político" (o más bien "micropolítico"), implicando al mismo tiempo la puesta en práctica de todo un conjunto de técnicas "manageriales" tomadas del ámbito expe-

16 A. J. Porras Nadales (1988), Introducción a una Teoría del Estado Postsocial, Barcelona, PPU.

17 A. J. Porras Nadales y M. Zafra Víctor (2002), El marco jurídico institucional de la acción pública, (Curso a distancia de Dirección y Gerencia Pública), Sevilla, IAAP. 
rimental de la gestión privada: innovaciones tecnológicas, organizativas y procedimentales, orientación estratégica, evaluación de resultados, etc. Todo este amplio proceso transformador acaba impactando inexorablemente con las inercias históricas adquiridas, tanto con la inercia del estado de partidos, desde la cual se entiende que todas las claves de la acción reposan sobre el circuito político-electoral legitimado por las urnas; como con la inercia de la burocracia tradicional, centrada en la mera aplicación de la ley y de las rutinas administrativas.

Pero no debe olvidarse que este impacto superador de los modelos pasados supone al mismo tiempo una vía operativa para dar respuesta a algunos de los principales desafíos del estado contemporáneo, que se enfrenta históricamente a dos diferentes fenomenologías de crisis: por una parte, la crisis del estado de bienestar o welfare state, entendida fundamentalmente como una crisis de eficacia en el cumplimiento de los horizontes finalistas que definen constitucionalmente al estado social; por otra, la crisis de legitimación del estado democrático avanzado, entendida básicamente como una insuficiencia del circuito representativo (y por lo tanto del sufragio universal) exigiendo la presencia adicional de procesos participativos en la toma de decisiones. En respuesta a la crisis de eficacia, se trataría pues de dinamizar y reformar la burocracia, concebida como el circuito instrumental de la acción estatal, descentralizando la gestión e introduciendo técnicas de gestión estratégica propias de las empresas privadas, con una evaluación constante de resultados. En respuesta a la crisis de legitimación, se trataría de incorporar los distintos circuitos participativos que, de una forma concentrada y a modo de redes sociales, operan en torno a los distintos ámbitos sectoriales de las políticas públicas: una participación que no tendrá tanto un carácter "directo" en sentido individual, sino que operará a través de los múltiples circuitos colectivos representativos de la sociedad organizada, implicando también la presencia del conocimiento experto. Surgiría así en definitiva el paradigma de la democracia eficaz, entendida como el objetivo estratégico hacia el cual se orienta la categoría de la moderna gobernanza.

\section{Andalucía: experiencias y antecedentes}

La pregunta inmediata sería en qué medida desdelas distintas Comunidades Autónomas, y en este caso desde Andalucía, se han dado o no condiciones de avance histórico hacia esta nueva línea de reformas, que deben traducirse en una mayor competitividad y eficacia de los aparatos públicos. Ya hemos 
apuntado que, en todo caso, las esferas regionales europeas se configuran en la actualidad como el principal campo estratégico para el desarrollo futuro del estado de bienestar ${ }^{18}$. Naturalmente no se trata de un conjunto de exigencias a las que las propias Comunidades puedan responder de una forma perfectamente autónoma, pues tanto la esfera del estado central como más recientemente la instancia europea, operan como circuitos condicionantes, a partir de los cuales se diseña el paradigma de la "gobernanza multinivel" como desafío del futuro ${ }^{19}$.

Sin embargo, parece claro que, al cabo de un cuarto de siglo, y pese a que el diseño organizativo originario se limitaba a reproducir el viejo modelo burocrático del estado centralista preexistente, hay que suponer que desde las Comunidades Autónomas se han puesto en marcha diversas experiencias de innovación en respuesta a necesidades emergentes o problemas sobrevenidos relacionados con el ejercicio de un sistema de competencias que se sitúa estratégicamente en el ámbito vital más inmediato de los ciudadanos, implicando el desarrollo de todo un conjunto de servicios y políticas propias del estado de bienestar avanzado.

En este sentido convendría comenzar recordando el dato histórico de que, en sus etapas iniciales, la Comunidad Autónoma de Andalucía manifestó un alto grado de beligerancia político-institucional en relación con el horizonte finalista originario, que se definía en el propio Estatuto en torno al objetivo de la lucha contra el subdesarrollo económico, implicando una intensa apuesta a favor de un sector público capaz de promover el desarrollo industrial en Andalucía. De ahí procede la singular experiencia del caso IPIA ${ }^{20}$ y de algunas de sus más espectaculares actuaciones bajo la dirección de Ricardo Sánchez de la Morena: hoy, sin embargo, el caso IPIA es probablemente más conocido en medios científico-académicos fuera de Andalucía, y sus pautas de actuación siguen siendo un dato singular de escaso impacto sobre las prácticas de la gestión pública andaluza.

18 A. J. Porras Nadales (1987), "Desarrollo autonómico y crisis delEstado social: el caso de Andalucía”, Revista Española de Investigaciones Sociológicas, nº 37; (1989), "Desarrollo y transformaciones jurídicas del Estado social en el modelo territorial autonómico español”, Revista del Centro de Estudios Constitucionales, nº 4, pp. 269-291.

19 A. J. Porras Nadales (2001), "Estado Postsocial y Unión Europea", en Aparicio, M. A., (coord.), Derechos Constitucionales y Formas Políticas. Actas del Congreso sobre derechos constitucionales y Estado Autonómico, Barcelona, Cedecs.

20 M. BARZELAY y J. Ma O’KEAN (1989), Gestión pública estratégica. Enfoques, análisis, experiencias. El caso IPIA, Madrid, Instituto de Estudios Fiscales. 
En segundo lugar, cabría aludir a las experiencias de lo que podríamos denominar como la "primera" oleada modernizadora de las administraciones públicas, que afecta en general a casi toda España desde finales de los ochenta hasta comienzos de la década final de siglo. Su iniciativa más destacada sería la de los puntos de información ciudadana, que se entendían como un instrumento de apertura y accesibilidad hacia los ciudadanos por parte de los aparatos públicos, en una línea de acción que generalmente se acompañaba de toda una serie de elementos cosméticos de información al ciudadano y de marketing público. Su balance general al cabo del tiempo resulta bastante tenue, en parte por falta de continuidad, en parte por su dimensión meramente externa o cosmética: es decir, que no incidía sobre los concretos sistemas organizativos y procesos decisionales de la administración autonómica ${ }^{21}$.

Un instrumento de mayor trascendencia sería el inicio de los primeros programas de calidad ${ }^{22}$, desde donde comienzan a incorporarse a la administración perspectivas mucho más sustanciales en términos de manejo y aplicación de la moderna cultura gestora; aun cuando el balance global de su implementación refleja finalmente logros limitados. Por último, la iniciativa de las ventanillas únicas u oficinas de respuesta unificada tuvo que esperar el impulso de algún proceso de concertación social para desencadenar su puesta en marcha, seguida de un itinerario de implementación relativamente lento, igualmente con un balance provisional que, hasta el presente, no refleja resultados espectaculares.

Como sucede con la valoración general que suscita esta llamada "primera modernización" de las administraciones públicas en España, el balance en Andalucía no resulta ser excesivamente satisfactorio: no ya por las conocidas críticas de focalización sobre ámbitos meramente cosméticos o de imagen, sino más probablemente por la sustancial carencia de un efecto dinamizador sobre el conjunto, de tal modo que la difusa inercia de la continuidad acaba predominando sobre los intentos localizados de innovación.

Hay sin embargo una experiencia organizativa sectorial que, al cabo del tiempo, viene reiterando una valoración positiva: nos referimos a la política

21 A. J. PoRRAS NADALES (1994), "La administración andaluza entre modernización y clientelismo", Autonomies, $\mathrm{n}^{\mathrm{O}}$ 18, pp. 81-91.

22 J. BARRAGÁn (2000), Un intento de concreción del paradigma de la nueva gestión pública. La pretensión de controlar la gestión de calidad en los servicios públicos de la Junta de Andalucía, Sevilla, IAAP. 
sanitaria, orquestada en torno a la dualidad entre las funciones finalistas y reguladoras de la Consejería y la función prestadora que asume el correspondiente Servicio Andaluz de Salud (o algunas empresas públicas) articuladas en torno a la figura de los contratos-programa. Se trata de una dinámica que, al cabo del tiempo, ha generado sus propias sinergias de retroalimentación e innovación que se mantienen de forma consistente: bien es cierto que se trata de un sector caracterizado por un intenso apoyo financiero (con efectos no siempre transparentes en términos de contabilidad pública) y una aceptación bastante escéptica por parte del sector profesional de médicos; debiendo tenerse en cuenta adicionalmente que el mismo tipo de modelo organizativo no ha tenido resultados igualmente positivos al aplicarse en otros sectores. Pero puede considerarse en todo caso una experiencia positiva susceptible de ser tomada en cuenta como ejemplo de referencia para el conjunto de la administración autonómica.

Un balance consistente de la experiencia de gestión pública en Andalucía nos ofrecería pues unos resultados finales bastante difusos, con una gran heterogeneidad entre distintos sectores que normalmente parecen afectados por un alto grado de desconocimiento recíproco, constatándose en general notables retrasos y deficiencias en los procesos de implementación, así como considerables riesgos difusos de no-acción. Las experiencias potencialmente originales de gestión o de innovación tienen en la práctica un escaso grado de proyección, debido seguramente a un déficit estructural de transversalidad generado por una estructura burocrática tradicional y verticalizada, bastante inmune a la transferencia de conocimientos y experiencias, y en general, al espíritu de innovación.

En definitiva, cabe considerar que, a pesar de que en Andalucía no han faltado durante las dos últimas décadas experiencias de programación al nivel de la agenda o de discurso político con un considerable desarrollo en forma de planes de actuación ${ }^{23}$, sin embargo la fase de implementación ha constituido desde entonces una gran tarea pendiente, tanto en lo que respecta a la fijación específica de objetivos y tareas para las distintas unidades de

23 Especialmente durante la década de los ochenta, cuando se formulan no sólo el objetivo programático de la lucha contra el subdesarrollo, sino los posteriores mitos de la vertebración, la modernización, e incluso la "California de Europa": todos ellos a partir de una notable integración de elementos reflexivos o de diagnóstico, y de estrategias finalistas o programáticas. Cfr. sobre el tema A. J. PoRRAS NADALES (1993), "El diseño de las políticas públicas en el contexto autonómico: el caso de Andalucía", Revista de Fomento Social, nº 191, vol. 48, pp. 359-381. Cfr. también Diagnosis y programación política, cit. 
las organizaciones públicas afectadas como, sobre todo, en lo que se refiere a su puesta en práctica y seguimiento efectivo a lo largo del tiempo de los distintos planes o programas (implicando pues una adecuada evaluación de los mismos). Una cierta dinámica perversa de "reinos de taifas" parece dificultar reiteradamente la proyección horizontal de sinergias y conocimientos, predominando la tradicional lógica vertical y compartimentalizada. El mantenimiento de una cultura burocrática tradicional, el uso de instrumentos jurídicos de carácter tradicional, y la reiterada persistencia de unos modelos arcaicos de organización, contribuyen a producir como efecto inmediato un reiterado déficit de implementación. La ausencia adicional de instrumentos adecuados de evaluación tiende a dificultar la percepción de los problemas así como los resultados de las distintas políticas y sus correspondientes planes de actuación, remitiendo en consecuencia su valoración general al evanescente mundo del discurso político.

\section{Andalucía ante los desafíos del futuro: la reforma de la Administración}

A comienzos del siglo XXI, y en el contexto general de reflexión histórica sobre el balance del proceso autonómico y sus perspectivas de reforma, se suscitan pues numerosos interrogantes que afectan a la operatividad y la eficacia de la acción pública autonómica en Andalucía. De entrada, cabría constatar algunos cambios recientes en la forma de abordar este problema, al menos en la medida en que podemos contar ya con la emergencia difusa de un nuevo tipo de conciencia o de "cultura" formada en el enfoque de las políticas públicas y en el paradigma de la nueva gestión pública, especialmente entre profesionales de la propia administración, así como en algunos ámbitos académicos universitarios: seguramente se trata todavía de un fenómeno algo difuso que, aunque no haya alcanzado un nivel suficiente de "masa crítica" desde una perspectiva científica, dispone ya sin embargo de un suficiente grado de conocimiento empírico y de prácticas de gestión acreditadas en el ámbito público. La propia expansión general de la literatura científica sobre el tema y sus abundantes traducciones en castellano, contribuyen a acercar este tipo de conocimiento a la propia realidad inmediata de los profesionales de la administración andaluza.

A ello se une el claro desafío programático procedente de la Unión Europea a partir de su Libro Blanco sobre la Gobernanza de 2001, implicando en consecuencia una clara reorientación de las pautas de acción pública, que debe afectar lógicamente a todo el entramado territorial europeo, y especialmente a las instancias regionales. 
Es difícil percibir si tales procesos de cambio estarían ya en condiciones de permitir la expansión práctica de una nueva cultura organizativa, con posibilidades de impacto generalizado sobre la gestión pública andaluza: parece evidente que las inercias del modelo burocrático dominante siguen manteniendo una fuerte presencia sobre la realidad cotidiana, al mismo tiempo que ciertas experiencias que en principio podrían considerarse como innovadoras, relacionadas con la actuación de determinadas empresas públicas, no han conseguido hasta ahora acreditarse como casos prácticos de valor paradigmático. Y tampoco el circuito político parece actuar hasta el presente conforme a una orientación unificada en este sentido.

Ahora bien, estos pasos limitados, que difusamente estarían señalando hacia la emergencia de nuevos paradigmas discursivos en torno a la gestión pública andaluza, se sitúan ahora ante un nuevo ambiente conectado con los desafíos programáticos de la llamada Segunda Modernización de Andalucía, donde se enfrenta la adecuación histórica de nuestra comunidad al impacto general de la revolución de las nuevas tecnologías de la información y el conocimiento (TIC). Bien es cierto que, en este ámbito, el impacto de las TIC en sí mismo considerado presenta algunas líneas de orientación que recuerdan la engañosa dimensión "mágica" del conocimiento tecnológico: el sueño de la e-administración que permitiría una gestión on-line de expedientes y de tramitaciones, de una forma perfectamente transparente e instantánea; un sueño que sin duda presenta una razonable dosis de congruencia para cierto tipo de tramitaciones directas con el ciudadano, pero que se limitaría al final a una serie de actuaciones de carácter periférico y de dimensión claramente cosmética en la medida en que los circuitos organizativos nucleares, desde donde se decide la programación intervencionista, se entienden como esferas ajenas que, en consecuencia, quedarían fuera de un eventual programa de reformas. Es evidente que la atención al ciudadano constituye una de las prioridades de toda reforma de la administración pública: pero también que la focalización exclusiva en este campo contribuye indirectamente a dejar fuera de la agenda los aspectos más sustantivos, y sin duda más procelosos, de toda política pública orientada a la reforma de la gestión pública.

Sin embargo parece que el sentido de la Segunda Modernización no se reduce exclusivamente a este aspecto más o menos superficial, de mero uso cuantitativo de las nuevas tecnologías, sino que se orienta hacia un cambio general en las mentalidades y por lo tanto en la concepción general de las pautas que deben orientar la acción pública en las sociedades democráticas del siglo XXI: en este sentido, parece que el debate se conecta con algunos 
otros procesos de reflexión puestos en marcha tanto en Andalucía como en otras Comunidades sobre el balance de las primeras etapas del desarrollo autonómico y la emergencia de nuevos desafíos relacionados con la configuración de los sistemas institucionales y el papel de las esferas públicas regionales ante un nuevo ambiente histórico que cuenta con el nuevo horizonte de la reforma de los Estatutos de Autonomía.

Si esta confluencia de coordenadas está abriendo una auténtica "ventana de oportunidad" para la puesta en marcha de una política pública de gestión pública en Andalucía, es algo que provisionalmente sólo cabe sugerir como mera hipótesis; al menos, mientras subsistan elementos inerciales y fuerzas conservadoras capaces de actuar como freno. Sin embargo, probablemente puede ser un momento adecuado para avanzar en la formulación de lo que deberían ser las principales líneas de actuación de un programa congruente de reformas. En este ámbito, la experiencia comparada ${ }^{24}$ nos señala como mínimo la presencia de tres o cuatro bloques estratégicos de acción: por una parte, el eje referido a la organización y a los métodos de funcionamiento; por otra, el que enfrenta la política de personal o recursos humanos; y finalmente, el fundamental capítulo de la gestión financiera.

En todos estos ámbitos se percibe en Andalucía, de forma diferenciada en cada caso, el diagnóstico común del déficit de implementación, consecuencia de una cultura organizativa de carácter tradicional donde las estructuras orgánicas se han venido ensanchando a lo largo del tiempo en un proceso perverso que podríamos denominar como una especie de "horizontalización hacia arriba" cuya consecuencia funcional más inmediata parece ser la progresiva "des-responsabilización" de las unidades, y sus efectos colaterales de ralentización e incluso de no-acción. Este modelo burocrático de carácter compartimentalizado contribuye adicionalmente a dificultar el flujo de experiencias o de conocimientos en el interior de la propia organización, configurando así un sistema de unidades estancas y fragmentarias, claramente inadecuado para encarar programas de carácter transversal.

Pues bien, en torno a estas diferentes áreas podría formularse, a partir del diagnóstico básico, un paquete mínimo de objetivos:

a) En relación con el área de organización y métodos parece evidente la necesidad de reformar las estructuras centrales, fundamentalmente

24 Citamos a partir del Proyecto de Investigación sobre "Public Management Policy Change", (2002), dirigido por el Prof. M. BARZELAY (London School of Economics). 
para atribuirles una mejor capacidad de dirección y de orientación finalista; al mismo tiempo que deben ampliarse los circuitos de prestación conforme a modelos de carácter horizontalizado y de estructura flexible, procediendo en general a la introducción de nuevos métodos de funcionamiento.

b) En relación con el área de recursos humanos, parece necesario avanzar en criterios de flexibilización que incidan en la excesiva rigidez del estatuto de los funcionarios, claramente inadecuado para una filosofía de organizaciones abiertas y dependientes del entorno; poniendo en marcha igualmente mecanismos capaces de asegurar una mayor motivación del personal público mediante un sistema de incentivos acorde con el funcionamiento demandado por los ciudadanos; así como enfrentar la fundamental tarea de conformar con claridad una función directiva profesionalizada.

c) Finalmente, el área de la gestión financiera requiere una urgente reforma orientada a acelerar los pagos, lo que implica probablemente la eliminación del pesado sistema centralizado de intervención previa y la introducción de nuevos medios más operativos de gestión presupuestaria.

Para encarar esta compleja serie de tareas se requiere partir de un núcleo argumentativo central que serviría como criterio de orientación estratégica, y que podemos deducir de las nuevas doctrinas sobre la gestión pública, en torno a una doble línea de reformas: por una parte, centralizar los circuitos de dirección; por otra, ampliar las bases prestadoras del sistema.

La primera línea implicaría reducir los servicios centrales de las Consejerías, para atribuirles exclusivamente funciones de carácter finalista o regulador, consistentes en asignar objetivos o definir tareas de carácter específico, estableciendo regulaciones de carácter general, así como controlar el cumplimiento de los mismos. Alternativamente, la segunda línea de respuesta consistiría en reordenar las esferas "periféricas" o de gestión, para ajustarlas a modelos prestadores de tipo horizontal y estructura flexible: los llamados "staffs" en línea.

La reducción de los servicios centrales permitiría adicionalmente integrar horizontalmente toda una serie de servicios que en la actualidad se repiten fragmentadamente en las estructuras verticales de las distintas Consejerías. Pero la novedad consistirá fundamentalmente en la nueva dimensión funcional que deben asumir estos servicios centrales: las tareas de definir fines, 
establecer regulaciones, elaborar instrumentos de planificación estratégica y fijar los standards de prestación de servicios; así como, por supuesto, la supervisión y control en el cumplimiento de los mismos. Son tareas todas ellas que deben ser asumidas por las estructuras centrales de las Consejerías. En clave organizativa, tal propuesta implica pues una reducción de los servicios centrales (el llamado "downsizing"), cuyo esquema organizativo se ajustará mejor a una ordenación por áreas, al mismo tiempo que se amplían los recursos transversales o comunes a diversos departamentos. En todo caso, la estructuración interna de estas áreas centrales se ajustaría a la dualidad entre sectores de planificación o regulación, frente a los de evaluación y control, buscando un modelo simple y clarificado de atribución de responsabilidades.

En cuanto a los circuitos de prestación, encargados directamente de las funciones de gestión e implementación de tareas y servicios, la experiencia adquirida hasta ahora nos demostraría la conveniencia de optar por un modelo flexible de agencialización pública: lo que deberá implicar la introducción de una filosofía emprenditorial en el sector público, orientada hacia la generación de "valores públicos" 25 . Serían estas agencias públicas las encargadas de llevar a cabo los objetivos asignados por la esfera central: nuevamente en clave de experiencia adquirida, debe recordarse la operatividad que, al cabo del tiempo, se ha venido demostrando en el sector de salud a través de la dualidad funcional Consejería-SAS, articulada a través de la figura de los contratos-programa.

En este contexto, el flujo de la acción se canalizaría de una forma directa y simplificada mediante la relación entre los servicios centrales asignadores de fines y tareas, y las agencias públicas prestadoras, encargadas de su efectivo cumplimiento: la práctica habitual del control y la evaluación permitiría la inmediata puesta en práctica de responsabilidades, en caso de incumplimiento de los objetivos asignados. Un sistema de agencias prestadoras de carácter público, que se podría poner en marcha de forma gradual, permitiría además la generalización progresiva de nuevos métodos gerenciales de acción, operando desde claves innovadoras, siguiendo en general el enfoque procedimental propio de las "políticas públicas": programación abierta de tareas, transparencia, flexibilidad organizativa, responsabilidad en la consecución de objetivos y evaluación periódica de resultados. Unas

25 En el sentido de M. H. Moore (1998), Gestión estratégica y creación de valor en el sector público, Barcelona, Paidos. 
exigencias de apertura y comunicación que se podrían proyectar no sólo a la propia organización del ejecutivo andaluz, sino también al Parlamento, asegurando así unos circuitos adecuados de control sobre la acción pública autonómica.

Esta pauta general de reordenación tendría igualmente su réplica en el sector de recursos humanos: la pesada política centralizada de personal debería ser sustituida por un modelo más flexible, donde la estructura organizativa y los recursos de cada unidad puedan adecuarse a la concreta estrategia de acción, y no a la inversa. Conforme a un marco legal que permitiera un sistema flexible de adscripción voluntaria, cabe suponer que un gran número de miembros del personal público (seguramente los más jóvenes y/o con mayor potencial de motivación profesional) estarían dispuestos a embarcarse en nuevas unidades agencializadas, donde su carrera profesional va a tener seguramente cauces más flexibles y operativos de desarrollo, en un marco ajustado a la normativa de contratación laboral.

La puesta en práctica de estos modelos de organización implicará complementariamente la introducción de nuevos métodos de funcionamiento. El eje de prioridad debe situarse lógicamente en torno a la tarea de fijación de objetivos y tareas, que debe entenderse como elemento necesario para el funcionamiento de cada unidad de gasto, teniendo un carácter pormenorizado. En la práctica existen ya numerosas experiencias en torno a las denominadas Cartas de Servicios, publicadas en distintas Comunidades Autónomas. Su reverso complementario serían los principios de autonomía y responsabilidad de las unidades de gestión: lo que debe entenderse como responsabilidad en cuanto a la consecución de los objetivos asignados; o sea, en términos de congruencia entre lo realmente producido y lo que se espera de tales organizaciones por parte de quienes van a supervisarlas. La unificación entre ambos elementos se completaría estratégicamente con la introducción generalizada de sistemas de evaluación de carácter externo, orientados a controlar tanto la eficiencia o adecuación al coste previsto, como la eficacia en términos de resultados sociales conseguidos: su aplicación implicará la puesta en marcha de la correspondiente responsabilidad gestora por parte del staff directivo.

En línea de funcionamiento interno, debe proponerse la sustitución del ancestral postulado de la opacidad burocrática por los de apertura y transparencia: tanto en términos de programación, como en relación con la proyección comunicativa con ciudadanos y redes sociales afectadas, así como con el resto de organismos públicos. Lo que implica entender la acción pública 
desde una perspectiva de apertura, con un flujo de comunicación abierto en primer lugar al propio personal público sobre la prestación de los servicios y la realización de tareas (aceptando pues las críticas constructivas del propio personal), y en un entorno de debate permanente con la red social afectada y con el entorno exterior. Adicionalmente, las áreas de gestión deben ajustarse al uso de nuevas técnicas de tipo gerencial, implicando la puesta en práctica de criterios de calidad (básicamente homologados en soportes tipo ISO), el uso del marketing como circuito de información y la generalización de las Cartas de Servicios entendidas no sólo como imagen de marca sino como un auténtico marco documental explícito que recoge los compromisos de la organización con sus clientes, garantizando así los derechos de los usuarios. En este sentido debe insistirse en que todas las tareas, además de ser sometidas a evaluaciones de rendimiento por unidades, deben diseñarse sobre la base de los compromisos efectivos que se adquieren por parte de la respectiva organización, concretando en su caso cuáles son las consecuencias para el usuario ante el incumplimiento de tales compromisos.

\section{El debate sobre la estrategia: oportunidades, obstáculos y posibilidades}

Es fácil entender que las perspectivas de éxito de una propuesta general de reforma de la administración no pueden depender exclusivamente de una simple cuestión de voluntad política: la puesta en marcha de una política pública de gestión pública que pretenda adquirir consistencia en el tiempo y generar resultados transformadores sustanciales requiere igualmente operar dentro de un ambiente social adecuado y actuar a través de una línea estratégica consistente. Aunque la primera clave de éxito dependerá del grado de consistencia del correspondiente impulso político: en este sentido, las coordenadas más idóneas serán normalmente las que se definen en el periodo inicial de una legislatura, es decir, el momento en que las expectativas colectivas comienzan a canalizarse desde la perspectiva de la función de dirección política, entendida como el marco finalista de partida en el que se define el compromiso originario de gobierno para un mandato parlamentario. Sin embargo, la lógica del mero impulso político necesitará adicionalmente de una orquestación estratégica e instrumental adecuada, que dependerá en gran medida del grado de compromiso activo que pueda conseguirse con la propia organización afectada. En otras palabras, el momento de la "política" (politics) deberá ir seguido del momento de las "políticas" (policies), implicando claves de compromiso y de consenso con la organización administrativa correspondiente. 
Desde una perspectiva estratégica suele afirmarse que, generalmente, un programa de reforma de la administración debe concentrarse en primer lugar en algunos puntos focales o centros de innovación, que actúen a modo de puntas de lanza, operando un efecto de arrastre sobre el resto de la organización: o sea, que la alternativa de un programa que pretenda ponerse en marcha de forma generalizada y simultánea para el conjunto de un sistema administrativo, corre el riesgo de quedar diluido ante las resistencias difusas de la organización burocrática y el mantenimiento de las inercias existentes. Deben ser esos puntos focales o centros de innovación los que actuarán como impulsores del proceso general de aprendizaje e innovación, impactando sobre el resto de las organizaciones afectadas como ejemplos inmediatos de "mejores prácticas".

Más difícil parece definir cuáles serían las variables de tipo "sociocultural" capaces de configurar un ambiente adecuado para el cambio: es decir, el tipo de cultura de la innovación que debería hacerse presente tanto en el entorno de la propia organización pública como en la periferia de la misma (es decir, en los medios académicos o incluso judiciales), así como, finalmente, en el ámbito exterior integrado por los propios ciudadanos-usuarios. Parece claro que en Andalucía, siguiendo la inercia del modelo europeo continental, predomina hasta ahora una cultura jurídica de tipo tradicional que se plasma en la correspondiente visión reduccionista de la administración entendida como una estructura burocrática adecuada a un soporte procedimental y rutinario de base legal y a unos controles jurisdiccionales de carácter tradicional: esta visión tópica, ajustada al viejo modelo "maxweberiano", hunde en parte sus raíces en el efecto de retraso histórico generado por el largo periodo de la dictadura franquista, así como en la larga inercia de dependencia de nuestro derecho público de la tradición napoleónica francesa. La concepción alternativa, que implicaría una visión de los instrumentos jurídicos como mecanismos de innovación de la realidad social, o como instrumentos para la gestión del cambio histórico, parece adolecer hasta ahora de una cierta debilidad, incluso entre medios científico-académicos que habitualmente se califican a sí mismos como "progresistas" ${ }^{26}$. Dejando a un lado la presencia de lógicos intereses corporativos, parece claro que predomina aquí la arcaica visión perversa de la administración entendida

26 Para un debate actualizado sobre las posibilidades de encuentro entre la gestión pública y el derecho administrativo europeo cfr. Instituto Europeo de Florencia: A. GEORGE, P. MACHADO y J. ZILLER (eds.), Law and Public Management, Working Papers (iue.it/PUB/WorkingPapers). 
como un potencial "enemigo" de los derechos ciudadanos, o como un foco de arbitrariedad y de opacidad: una concepción tras la que frecuentemente se esconden intereses de sectores sociales dominantes. Es decir, la idea de una administración entendida como "caja negra" sólo accesible a determinados intereses "consolidados" (modelo que difícilmente se ajusta a las claves de transparencia y participación propias de las democracias avanzadas) implicando en consecuencia una posición de la instancia jurídica como instrumento de control o de freno sobre la misma, y no como un medio para asegurar su desarrollo innovador y su mayor eficacia. Por supuesto, se trata de un reduccionismo que no responde en absoluto a exigencias de tipo constitucional, pues el paradigma del Estado de Derecho supone efectivamente la sujeción de la administración a la ley en general, pero no a un determinado modelo de ley "administrativa".

Ciertamente, este tipo de mentalidad dominante hace tiempo que parece haber comenzado a cambiar, especialmente en el entorno del propio personal público, es decir, entre quienes tienen que "luchar" diariamente contra las limitaciones y los constreñimientos de un sistema jurídico-procedimental estrecho y falto de operatividad e idoneidad para el logro de objetivos concretos por parte del sector público. Sin embargo, paradójicamente, la mentalidad tradicional subsiste entre el entorno profesional y académico externo, así como en numerosos sectores del personal judicial.

Dos serían las líneas argumentativas que, en clave jurídica, parecen justificar la permanencia en el tiempo de este tipo de mentalidad tradicional entre sectores profesionales del medio jurídico: por una parte el fenómeno (entendido como una especie de amenaza) de la denominada "huida del derecho administrativo", por otro el de la potencial afectación de derechos fundamentales de los ciudadanos. Se trata de un doble argumento que se monta sobre una paralela serie de falacias: por una parte, la confusión entre la huida del derecho administrativo y la pura privatización o desregulación; por otra, el espejismo de que los derechos fundamentales de los ciudadanos son "mejor" defendidos por un arcaico sistema de procedimiento administrativo que por un modelo actualizado y transparente de gestión pública estratégica.

Por supuesto, debe aceptarse que la falta de un desarrollo consistente de las iniciativas de innovación puestas en marcha hasta ahora en la organización pública andaluza, ha generado efectivamente algunas experiencias perversas que justifican sobradamente las numerosas suspicacias existentes en el medio social o académico: algunos supuestos de diseño institucional y 
agencialización se han conformado al servicio de específicos intereses políticos o corporativos, sin contar con un modelo de referencia bien definido, y generando una creciente opacidad en los sistemas de visualización y control de la acción pública. Del mismo modo que reiteradas prácticas de externalización se han llevado a cabo de forma difusa y más o menos descontrolada. Se trata de todo un conjunto de actuaciones que no constituyen en absoluto el tipo de "buenas prácticas" sobre las que debería montarse un proceso consistente de reformas de la organización pública andaluza.

Un proceso de agencialización congruente exigiría lógicamente el diseño legal de un estatuto básico de las agencias públicas, implicando el reconocimiento de los derechos de los ciudadanos-usuarios y la apertura de mecanismos de control de carácter transparente, proyectables incluso hacia el propio parlamento autonómico. Se trata de un campo donde el uso de las nuevas tecnologías debe permitir una más fácil instrumentación de las exigencias de transparencia, superando así la tradicional opacidad de la burocracia tradicional. Y naturalmente, donde el tipo de derechos ciudadanos que deben ser garantizados no se limitaría a la mera posición pasiva del "administrado" individual, sino que debería diseñarse sobre la configuración de una red social de ciudadanos-usuarios (las denominadas en la literatura especializada como "policy communities") dotados de una pretensión de presencia activa en el proceso general de la gestión y la acción, tanto en su fase de implementación como de evaluación.

Desde los principales centros de producción y reproducción de la cultura jurídica de Andalucía, tanto académicos como profesionales, debería pues enfrentarse la tarea de dilucidar el papel histórico que deben desempeñar las instancias jurídicas ante este desafío histórico: si la tradicional función de reproducción de pautas adquiridas y, por lo tanto, de freno a las innovaciones, o la función alternativa de liderar el cambio y convertirse en un motor de la innovación de los sistemas públicos de Andalucía. Es decir, se trata de enfrentar nada menos que un cambio del paradigma cultural dentro de un ambiente social complejo donde deberá darse por aceptado el mantenimiento de trincheras de resistencia frente a la cultura de la innovación y a las nuevas fronteras del derecho público.

Naturalmente las resistencias al cambio histórico pueden superarse siempre a base de apoyo político y de concertación entre las fuerzas sociales favorables al mismo: aunque en este sentido debe recordarse que la dinámica histórica por la que ha discurrido la concertación social en Andalucía ha acabado formalizando un proceso relativamente reduccionista, donde 
son exclusivamente los tradicionales interlocutores "sociales" (es decir, patronal y sindicatos) los protagonistas de un tipo de acuerdos que suelen tener numerosas cláusulas ocultas tras las que se esconden mecanismos de intercambio recíproco. Un programa de reforma global de la administración debería necesariamente ampliar el circuito de compromisos sociales, orquestando sus redes más allá del reducido círculo de los tradicionales interlocutores sociales, operando así como una auténtica política pública de gestión pública proyectada tanto hacia la organización pública como hacia el conjunto del sistema social.

\section{Consideraciones finales}

Las dificultades que un ambiente poco propicio pueda suscitar ante una política pública de reforma de la administración y de la gestión pública andaluza, no permiten sin embargo ocultar las innegables ventajas y oportunidades que, ante los nuevos desafíos que parece abrir el proceso de innovaciones conocido bajo la denominación de Segunda Modernización, tendría una iniciativa decidida de modificación modernizadora de nuestra Administración. Es evidente en primer lugar que existe una fuerte presión social difusa a favor de la reforma de nuestros aparatos públicos, muchos de ellos inmersos en una dinámica de inercia y de rutina que indirectamente conduce hacia el camino perverso de la "no-acción". Igualmente parece que la esfera política tendría siempre un camino más fácil a la hora de incidir sobre la propia organización pública, frente a la alternativa de otro tipo de políticas, siempre más complejas, que tratan de incidir sobre el tejido social ${ }^{27}$ : subyace en este ámbito probablemente un desafío de credibilidad que afectaría a la propia apuesta histórica por la Segunda Modernización de Andalucía.

Pero hay también toda otra serie de factores que incidirían de forma directa a favor de esta decisiva apuesta histórica: en primer lugar, el impacto difuso e inmaterial de las nuevas tecnologías, que suscitan siempre la expectativa de nuevas formas de diálogo e interacción entre los ciudadanos y la esfera pública. En segundo lugar, debe recordarse que no se trata exactamente

27 Una dualidad que parece estar presente en la valoración general del proceso de implementación de las competencias autonómicas durante sus primeras dos décadas. Cfr. sobre el tema: A. PorRAS y M. ZAFRA (2003), "La implementación de las competencias de la Junta de Andalucía: un balance", en A. J. PorRAs NADAles (coord), El sistema de competencias de la Junta de Andalucía y su desarrollo efectivo, (ed. XX Aniversario Estatuto de Andalucía), Granada, Comares, Parlamento de Andalucía. 
de operar una innovación radical del conjunto del sistema, sino más bien de avanzar sobre un terreno donde ya se han venido dando pasos sustanciales, tanto en términos de desarrollo de programas de calidad como en iniciativas agencializadoras (algunas de ellas con resultados francamente positivos); eso nos permite, a estas alturas, disponer de un balance experimental adecuado capaz de reducir los factores de incertidumbre. Se trataría pues de asegurar factores de impulso adicional sobre estos elementos de innovación para permitir que, en un marco regulativo general adecuado al nuevo contexto, desarrollen todas sus potencialidades de expansión sobre el resto de la organización.

Pero, sobre todo, debería insistirse en la noción de "cambio cualitativo" que implica la propuesta: es decir, no se trata tanto de "poner más dinero" para reformar la administración, sino de modificar pautas generales de organización y de funcionamiento, con el objetivo de asegurar una mayor eficacia y competitividad de nuestros aparatos públicos, en el entendimiento de que una administración que funcione mejor no tiene por qué ser necesariamente una administración más cara. De lo que se trata sería más bien de superar las viejas inercias adquiridas y los lastres históricos generados tras una primera etapa de desarrollo autonómico en la que se "importó" a la esfera autonómica toda una estructura (y una mentalidad) burocrática tradicional, que se ha ido reproduciendo a lo largo del tiempo, configurando así una noción reduccionista de la administración pública entendida como una instancia limitada a la mera "gestión de las competencias" transferidas: por el contrario, frente a la mera gestión de competencias, debemos reivindicar un modelo de administración con capacidad para diseñar políticas públicas propias, ajustadas al tipo de demandas y necesidades emergentes de la sociedad andaluza, adecuando la propia organización pública al servicio de los fines y los objetivos estratégicos que exige el nuevo contexto histórico.

La dimensión de estos desafíos a los que se enfrenta el sector público andaluz no permite seguramente dilatar por más tiempo la fundamental tarea de poner en marcha un programa de reformas que sea capaz de articular una auténtica política pública de gestión pública: máxime si tenemos en cuenta el nuevo contexto de competitividad que va a suscitar el proceso de expansión de la Unión Europea. Las exigencias de la nueva gobernanza se acaban proyectando inexorablemente sobre el tejido regional europeo, desde donde deben enfrentarse los decisivos problemas históricos que impone la reordenación territorial del estado social contemporáneo. 